\title{
Gaussian Barebones Differential Evolution with Random-type Gaussian Mutation Strategy
}

\author{
Hung-Ching Lü ${ }^{1}$, Ming-Feng Yeh ${ }^{2, *}$, Yu-Wei Lu ${ }^{1}$ \\ ${ }^{1}$ Department of Electrical Engineering, Tatung University, Taiwan \\ ${ }^{2}$ Department of Electrical Engineering, Lunghwa University of Science and Technology, Taiwan
}

Copyright $\mathrm{O} 2016$ by authors, all rights reserved. Authors agree that this article remains permanently open access under the terms of the Creative Commons Attribution License 4.0 International License

\begin{abstract}
This study attempts to propose a random-type Gaussian mutation strategy to improve the solution accuracy of Gaussian barebones differential evolution (GBDE). The proposed Gaussian mutation strategy is not only parameter free, but also employed to enhance the population diversity and global searching ability of the original mutation strategy. The search performance of GBDE with the proposed mutation strategy is compared with two standard DEs (DE/rand/1 and DE/best/1), the original GBDE and its modified version in terms of solution accuracy. Simulation results on two real-world optimal control problems given in IEEE - CEC 2011 evolutionary algorithm competition demonstrate the effectiveness of the proposed GBDE algorithm.
\end{abstract}

Keywords Differential Evolution, Mutation Strategy, Gaussian Sampling, Gaussian Barebones Differential Evolution

\section{Introduction}

Differential evolution (DE) algorithm, proposed by Storn and Price in 1995, is a simple yet powerful evolutionary algorithm (EA) for global optimization problems in the continuous search domain [1]. The performance of DE algorithm is generally sensitive to the mutation strategy and the respective control parameters such as the scale factor $F$, the crossover rate $\mathrm{Cr}$, and the population size $N p$ [2]. In order to minimize the effects of control parameters, a number of adaptive DE variants have been proposed in the past several years [3]-[5]. In addition, rather than using complex adaptive or self-adaptive mechanisms, barebones DE (BBDE) [6] and Gaussian barebones DE (GBDE) [7] are two almost parameter free DE variants. Both of them could achieve a good balance between exploration and exploitation in the search process.

GBDE is based on the Gaussian sampling. As indicated in [7], the Gaussian sampling is a fine tuning procedure which starts during explorations and is continued to exploitation. This can be beneficial for the search of many evolutionary optimization algorithms. The Gaussian mutation strategy is implemented by a Gaussian random function with mean $\mu=\left(\mathbf{x}_{\text {best }, G}+\mathbf{x}_{i, G}\right) / 2$ and standard deviation $\sigma=\left|\mathbf{x}_{\text {best }, G}-\mathbf{x}_{i, G}\right|$, where $\mathbf{x}_{\text {best }, G}$ and $\mathbf{x}_{i, G}$ represent the best vector and the $i$-th target vector at the $G$-th generation, respectively. GBDE also employs the crossover schemes used in the classical DEs. Without loss of generality, this study only considers the binomial crossover scheme. In [7], Wang et al also proposed the hybridization of Gaussian mutation and DE/best/1 to balance the global search ability and convergence rate. Such a GBDE variant is termed the modified GBDE (MGBDE). In MGBDE, each vector is randomly assigned a mutation strategy (either Gaussian mutation or DE/best/1) during the population initialization. The assigned mutation strategy for each vector does not change during the search process. About $50 \%$ of the vectors follow the behavior of $\mathrm{DE} / \mathrm{best} / 1$, while the remaining $50 \%$ follow the GBDE.

Gaussian mutation strategy is a kind of the best-so-far solution-based strategies. That is, the best solution will guide the direction of evolutionary search, and then might lead to the problem of premature convergence caused by the reduced population diversity. Among the classic DE strategies, "DE/rand/1" strategy, which is usually less greedy, has slower convergence speed, and is more reliable than the strategies based on the best-so-far solution $[1,5,8]$. This gives rise to the motivation to apply "DE/rand/1" strategy to Gaussian mutation strategy to propose a newly mutation strategy termed the random-type Gaussian mutation strategy. The newly developed random-type Gaussian mutation strategy is attempted to preserve the diversity of the population and the problem of premature convergence can be alleviated.

In order to verify the performance of the proposed Gaussian mutation strategy, the proposed strategy is applied to solve two real-word optimal control problems given in IEEE - CEC 2011 evolutionary algorithm competition [9]. The performance of the proposed strategy compares 
favorably with the original GBDE and MGBDE as well as two classical DEs, "DE/rand/1" and "DE/best/1".

The remainder of this paper is organized as follows. Section 2 briefly describes GBDE and its modified version MGBDE. The proposed random-type Gaussian mutation strategy is described in Section 3. Section 4 presents the search performance of the proposed approaches on two optimal control problems. Finally, Section 5 contains some conclusions of this study.

\section{DE Algorithms, GBDE and MGBDE}

\subsection{DE Algorithms}

Like other EAs, DE algorithm starts with a randomly generated initial population vector within the search space. After initialization, DE then enters a loop of evolutionary operations: mutation, crossover, and selection as follows $[1,4]$. Let $\mathbf{x}_{i, G}=\left(x_{1 i, \mathrm{G}}, x_{2 \mathrm{i}, \mathrm{G}}, \ldots, x_{D i, G}\right)$ be a target (solution) vector in generation $G$, where $i=1,2, \ldots, N_{P}\left(N_{p}=\right.$ population size).

Mutation: For each target vector $\mathbf{x}_{i, G}$, DE employs the mutation operation to produce a mutant vector $\mathbf{v}_{i, G}$. The following strategies are five most frequently used schemes:

$\mathrm{DE} / \mathrm{rand} / 1$ :

$$
\mathbf{v}_{i, G}=\mathbf{x}_{r 1, G}+F\left(\mathbf{x}_{r 2, G}-\mathbf{x}_{r 3, G}\right)
$$

DE/best/1:

$$
\mathbf{v}_{i, G}=\mathbf{x}_{\text {best }, G}+F\left(\mathbf{x}_{r 1, G}-\mathbf{x}_{r 2, G}\right.
$$

DE/current-to-best/1:

$$
\mathbf{v}_{i, G}=\mathbf{x}_{i, G}+F\left(\mathbf{x}_{\text {best }, G}-\mathbf{x}_{i, G}\right)+F\left(\mathbf{x}_{r 1, G}-\mathbf{x}_{r 2, G}\right)
$$

$\mathrm{DE} / \mathrm{rand} / 2$ :

$$
\mathbf{v}_{i, G}=\mathbf{x}_{r 1, G}+F\left(\mathbf{x}_{r 2, G}-\mathbf{x}_{r 3, G}\right)+F\left(\mathbf{x}_{r 4, G}-\mathbf{x}_{r 5, G}\right)
$$

$\mathrm{DE} / \mathrm{best} / 2$ :

$$
\mathbf{v}_{i, G}=\mathbf{x}_{\text {best }, G}+F\left(\mathbf{x}_{r 1, G}-\mathbf{x}_{r 2, G}\right)+F\left(\mathbf{x}_{r 4, G}-\mathbf{x}_{r 5, G}\right)
$$

where the indices $r 1, r 2, r 3, r 4$, and $r 5$ are distinct integers randomly generated from the set $\left\{1,2, \ldots, N_{p}\right\} \backslash\{i\}$, $\left(\mathbf{x}_{r 1, G}-\mathbf{x}_{r 2, G}\right)$ or $\left(\mathbf{x}_{r 2, G}-\mathbf{x}_{r 3, G}\right)$ is a difference vector to mutate the base vector, $\mathbf{x}_{\text {best }, G}$ represents the best vector at the $G$-th generation, and the parameter $F$ is called the scaling factor.

Crossover: Crossover operation is applied to combine the target vector $\mathbf{x}_{i, G}$ and its corresponding mutant vector $\mathbf{v}_{i, G}$ to generate a trial vector $\mathbf{u}_{i, G}=\left(u_{1 i, \mathrm{G}}, u_{2 \mathrm{i}, \mathrm{G}}, \ldots, u_{D i, G}\right)$. The widely-used binomial crossover scheme is defined as follows.

$$
u_{j i, G}=\left\{\begin{array}{lc}
v_{j i, G}, & \text { if }\left(\operatorname{rand}_{j} \leq C r\right) \text { or }\left(j=j_{\text {rand }}\right) \\
x_{j i, G}, & \text { otherwise }
\end{array}\right.
$$

for $j=1,2, \ldots, D$, where rand $_{j}$ is a randomly chosen real number within the range of $(0,1), C r$ is the crossover rate and $j_{\text {rand }} \in\{1,2, \ldots, D\}$ is a randomly chosen index which ensures that the trial vector $\mathbf{u}_{i, G}$ gets at least one element from $\mathbf{v}_{i, G}$.

Selection: The selection operation selects the better one from the target vector $\mathbf{x}_{i, G}$ and the trial vector $\mathbf{u}_{i, G}$ according to their fitness values. That is

$$
\mathbf{x}_{i, G+1}=\left\{\begin{array}{cc}
\mathbf{u}_{i, G}, & \text { if } f\left(\mathbf{u}_{i, G}\right) \leq f\left(\mathbf{x}_{i, G}\right) \\
\mathbf{x}_{i, G}, & \text { otherwise }
\end{array}\right.
$$

Using this greedy selection scheme, all individuals of the next generation are better than the individuals of the current population.

\subsection{GBDE and MGBDE}

In the GBDE, the Gaussian mutation strategy is defined as follows.

$$
\mathbf{v}_{i, G}=N(\mu, \sigma)
$$

where $N(\mu, \sigma)$ is a Gaussian random function and the corresponding mean $\mu$ and standard deviation $\sigma$ are defined as follows [7].

$$
\begin{gathered}
\mu=\left(\mathbf{x}_{\text {best }, G}+\mathbf{x}_{i, G}\right) / 2 \\
\sigma=\left|\mathbf{x}_{\text {best }, G}-\mathbf{x}_{i, G}\right|
\end{gathered}
$$

Similar to the classical DE, GBDE also employs the binomial crossover scheme (6). However, rather than using a fixed value of crossover rate, GBDE utilizes a simple self-adaptive strategy to dynamically update the crossover rate as follows.

$$
C r_{i, G+1}=\left\{\begin{array}{cc}
C r_{i, G}, & \text { if } f\left(\mathbf{u}_{i, G}\right) \leq f\left(\mathbf{x}_{i, G}\right) \\
N(0.5,0.1), & \text { otherwise }
\end{array}\right.
$$

where $N(0.5,0.1)$ is a random value generated by a normal function with mean 0.5 and standard deviation 0.1 .

The main steps of GBDE are described as follows.

Step 1: Determine the control parameters such as the scale factor $F$, the crossover rate $\mathrm{Cr}$, the population size $N_{p}$ and the maximum number of generations $G_{\max }$.

Step 2: Randomly initialize all the target vectors in the search space and set the generation count $G=1$.

Step 3: Determine the best vector $\mathbf{x}_{\text {best }, G}$.

Step 4: Mutation: Generate a mutant vector $\mathbf{v}_{i, G}$ by (8) with mean $\mu$ (9a) and standard deviation $\sigma$ (9b).

Step 5: Crossover: Generate a trial vector $\mathbf{u}_{i, G}$ by (6).

Step 6: Selection: Select the target vector for the next generation, $\mathbf{x}_{i, G+1}$, by (7).

Step 7: Update the crossover rate $\mathrm{Cr}$ by (10).

Step 8: Increment the generation count $G=G+1$.

Step 9: Repeat steps 3-8 until a stopping criterion is fulfilled (e.g., the maximum number of generations or the goal is reached).

In the MGBDE, each vector is randomly assigned a mutation strategy (either Gaussian mutation or DE/best/1) during the population initialization. The assigned mutation strategy for each vector does not change during the search process. Let $M_{i}$ represent the mutation strategy of $\mathbf{x}_{i}$. Then 
it can be expressed as follows.

$$
M_{i}=\left\{\begin{array}{cc}
\text { DE/best } / 1, & \text { if } \operatorname{rand}_{i}(0,1) \leq 0.5 \\
\text { Gaussian mutation, } & \text { otherwise }
\end{array}\right.
$$

For each vector $\mathbf{x}_{i}$, MGBDE first checks the $M_{i}$ and selects a corresponding mutation strategy to generate the mutant vector. The rest of the steps of the MGBDE are the same as that with GBDE.

\section{GBDE with Random-type Gaussian Mutation Strategy}

\subsection{Random-type Gaussian Mutation Strategy}

The proposed random-type Gaussian mutation strategy is also in the form of (8), but the corresponding mean $\mu$ and standard deviation $\sigma$ are obtained by

$$
\begin{aligned}
\mu & =\left[X_{r 1, G}+\frac{X_{r 2, G}+X_{r 2, G}}{2}\right] / 2 \\
\sigma & =\left|X_{r 1, G}-\frac{X_{r 2, G}+X_{r 2, G}}{2}\right|
\end{aligned}
$$

where the randomly chosen target vector $\mathbf{x}_{r 1}$ in (12a) is served as the base vector as that given in "DE/rand/1" mutation strategy. In addition, the average term $\left(X_{r 2, G}+\right.$ $\left.X_{r 2, G}\right) / 2$ plays the role of perturbation to help the base vector $\mathbf{x}_{r 1}$ move out of the local area as the term $\left(\mathbf{x}_{r 2, G}-\mathbf{x}_{r 3, G}\right)$ given in (1).

Compared (12a) with (9a) and (12b) with (9b), it can be seen that the best vector $\mathbf{x}_{\text {best }}$ corresponds to a randomly chosen vector $\mathbf{x}_{r 1}$, while the target vector $\mathbf{x}_{i}$ to the average of $\mathbf{x}_{r 2}$ and $\mathbf{x}_{r 3}$. The randomly chosen base vector $\mathbf{x}_{r 1}$ generally has better exploration capability than the best-based vector $\mathbf{x}_{\text {best }}$. Also the average term $\left(X_{r 2, G}+\right.$ $\left.X_{r 2, G}\right) / 2$ may result in better perturbation than the single target vector $\mathbf{x}_{i}$ as in the original Gaussian mutation strategy. Thus those modifications are employed to enhance the population diversity and global searching ability of the orignal Gaussiant mutation strategy. In addition, the proposed random-type Gaussian mutation strategy is also parameter free.

\subsection{Procedure}

As indicated in [7], MGBDE could achieve better performance than GBDE on many unimodal and multimodal functions. Therefore the following steps only discuss the procedure used for implementing the MGBDE with random-type Gaussian mutation strategy.

Step 1: Determine the control parameters such as the scale factor $F$, the crossover rate $C r$, the population size $N_{p}$ and the maximum number of generations $G_{\max }$.

Step 2: Randomly initialize all the target vectors in the search space and set the generation count $G=1$.

Step 3: Randomly assign a mutation strategy to each vector according (11).
Step 4: Determine the best vector $\mathbf{x}_{\text {best, } G}$.

Step 5: Mutation: Generate a mutant vector $\mathbf{v}_{i, G}$ according to the mutation strategy determined by Step 3. If "DE/best/1" is assigned, $\mathbf{v}_{i, G}$ is generated by (2), otherwise, $\mathbf{v}_{i, G}$ is generated by (8) with mean $\mu$ (12a) and standard deviation $\sigma(12 b)$.

Step 6: Crossover: Generate a trial vector $\mathbf{u}_{i, G}$ by (6).

Step 7: Selection: Select the target vector for the next generation, $\mathbf{x}_{i, G+1}$, by (7).

Step 8: Update the crossover rate $\mathrm{Cr}$ by (10).

Step 9: Increment the generation count $G=G+1$.

Step 10: Repeat steps 4-9 until a stopping criterion is fulfilled (e.g., the maximum number of generations or the goal is reached).

\section{Simulation Results}

In order to demonstrate the search performance of the proposed Gaussian mutation strategy, two real-world optimal control problems, viz., the bifunctional catalyst blend optimal control problem and the optimal control of a nonlinear stirred tank reactor [9], are used to verify it. The search performance of the proposed MGBDE variant (denoted as MGBDE/rand hereafter) is compared with two classical DEs (DE/rand/1 with $F=0.5$ and $C r=0.5$, and DE/best $/ 1$ with $F=0.5$ and $\mathrm{Cr}=0.5$ ), the original GBDE and its modified version MGBDE (denoted as MGBDE/bst-cur) in terms of solution accuracy In addition, this study also uses the Friedman test [10] and the Wilcoxon's signed rank test at $\alpha=0.05$ [11] to compare the performance of multiple algorithms on the test suite.

In the simulations, all the DE variants were tested using the same population size of 100 . Each algorithm stops when the number of fitness evaluations (FEs) reaches the maximum FEs of $1.50 \mathrm{E}+05$. Also each algorithm independently runs 25 times per function. All the programs coded by Matlab version R2010a were executed by a personal computer with Intel(R) Core(TM) i7-3770 CPU @ 3.40/3.40 GHz processor, 4.0-GB RAM and Windows 7 with service pack 1 operating system

\subsection{Problem Statements}

\subsubsection{The Bifunctional Catalyst Blend Optimal Control Problem}

The bifunctional catalyst blend optimal control problem is a chemical process which converts methylcyclopentane to benzene in a tubular reactor and described by a set of following seven differential equations $[12,13]$ :

$$
\begin{gathered}
\dot{x}_{1}=-k_{1} x_{1} \\
\dot{x}_{2}=k_{1} x_{1}-\left(k_{2}+k_{3}\right) x_{2}+k_{4} x_{5} \\
\dot{x}_{3}=k_{2} x_{2} \\
\dot{x}_{4}=-k_{6} x_{4}+k_{5} x_{5}
\end{gathered}
$$




$$
\begin{gathered}
\dot{x}_{5}=-k_{3} x_{2}+k_{6} x_{4}-\left(k_{4}+k_{5}+k_{8}+k_{9}\right) x_{5} \\
+k_{7} x_{6}+k_{10} x_{7} \\
\dot{x}_{6}=k_{8} x_{5}-k_{7} x_{6} \\
\dot{x}_{7}=k_{9} x_{5}-k_{10} x_{7}
\end{gathered}
$$

where $x_{i}, i=1,2, \ldots, 7$, are the mole fractions of the chemical species and the rate constants $k_{j}$ are cubic functions of the catalyst blend $u(t)$ :

$$
k_{j}=c_{j 1}+c_{j 2} u+c_{j 3} u^{2}+c_{j 4} u^{3}, j=1,2, \ldots, 10,
$$

where the values of the coefficients $c_{j l}, l=1,2, \ldots, 4$, are experimentally evaluated are given in [9]. The mass fraction of the hydrogenation catalyst is bounded as $0.6 \leq u(t) \leq$ 0.9 and the initial state is given as $(0)=$ $\left[\begin{array}{lllllll}1 & 0 & 0 & 0 & 0 & 0 & 0\end{array}\right]^{T}$.

Luus [10] indicated that this chemical process is operated in steady state. The state $x_{1}$ appears at the beginning of the process and $x_{7}$ at the end. Therefore $x_{1}$ through $x_{7}$ can be considered to be placed along the length of the tubular reactor. The objective is to determine the catalyst blend $u$ along the length of the reactor at time interval $0 \leq t \leq t_{f}$, where $t_{f}=2000 \mathrm{gm} / \mathrm{mol}$, such that the performance index

$$
J=x_{7}\left(t_{f}\right) \times 10^{3}
$$

representing the benzene concentration at the exit of the reactor, is maximized.

\subsubsection{Optimal Control of a Nonlinear Stirred Tank Reactor}

The optimal control of a nonlinear stirred tank reactor problem is a benchmark optimization problem in the handbook [14]. This chemical process is modeled by the following two nonlinear differential equations:

$$
\begin{gathered}
\dot{x}_{1}=-(2+u)\left(x_{1}+0.25\right)+\left(x_{2}+0.5\right) \exp \left(\frac{25 x_{1}}{x_{1}+2}\right) \\
\dot{x}_{2}=0.5-x_{2}-\left(x_{2}+0.5\right) \exp \left(\frac{25 x_{1}}{x_{1}+2}\right)
\end{gathered}
$$

where $u$ is the flow rate of the cooling fluid, $x_{1}$ is the dimensionless steady state temperature, and $x_{2}$ is the deviation from dimensionless steady state concentration. The optimization objective is to determine suitable value of $u$ so that the performance index

$$
J=\int_{0}^{t_{f}=0.72}\left(x_{1}^{2}+x_{2}^{2}+0.1 u^{2}\right) d t
$$

is minimizes with the initial conditions being $x(0)=[0.09$ $0.09]^{T}$ and the initial guess of $u(0)$ within $[0.05 .0]$.

\subsection{Comparison on Solution Accuracy}

The results obtained by DE/rand/1, DE/best/1, GBDE, MGBDE/bst-cur and MGBDE/rand are summarized in Table 1 in terms of solution accuracy (the mean of the best-of-run values for 25 runs). Table 1 also uses the Friedman test [10] to compare the performance of multiple algorithms on the selected control problems. The Friedman test ranks the algorithms for each problem separately, the best performing algorithm should have the rank of 1 , the second best rank 2, etc. In case of ties, average ranks are computed. In the table, the number given in the parentheses represents the corresponding Friedman rank. The last row of Table 1 also shows the Friedman average rank (FAR) of each DE variant. In Table 1, the best rankings are shown in boldface. As can be seen, MGBDE/rand with FAR of 2.0 outperforms MGBDE/bst-cur with FAR of 2.5 The fact reveals that the proposed random-type Gaussian mutation could improve the solution accuracy. MGBDE/rand also performs better than other three DE variants (GBDE, $\mathrm{DE} /$ best/1 and DE/rand/1).

In this study, the Wilcoxon's rank sum test [11] at $\alpha=$ 0.05 is also adopted to evaluate the statistical significance of the results. The comparison results among MGBDE/rand and other algorithms are summarized below the corresponding Friedman rank, where "+", "-", and "=" indicate MGBDE/rand is respectively better than, worse than, or similar to the corresponding algorithm according to the

\begin{tabular}{|c|c|c|c|c|c|c|c|c|c|c|c|}
\hline \multirow{3}{*}{$\begin{array}{l}4.1 .1 \\
\text { (T03) }\end{array}$} & \multirow[b]{2}{*}{ Mean } & \multicolumn{2}{|c|}{$\mathrm{DE} / \mathrm{rand} / 1$} & \multicolumn{2}{|c|}{$\mathrm{DE} /$ best $/ 1$} & \multicolumn{2}{|l|}{ GBDE } & \multicolumn{2}{|c|}{ MGBDE/bst-cur } & \multicolumn{2}{|c|}{ MGBDE/rand } \\
\hline & & $1.1545 \mathrm{E}-05$ & $(5.0)$ & $1.1531 \mathrm{E}-05$ & $(4.0)$ & $1.1524 \mathrm{E}-05$ & $(2.0)$ & $1.1525 \mathrm{E}-05$ & $(3.0)$ & $1.1523 E-05$ & (1.0) \\
\hline & St. Dev. & $5.8725 \mathrm{E}-08$ & $=$ & $3.1611 \mathrm{E}-08$ & $=$ & $1.2461 \mathrm{E}-08$ & $=$ & $1.7668 \mathrm{E}-08$ & $=$ & $1.5350 \mathrm{E}-08$ & --- \\
\hline \multirow{2}{*}{$\begin{array}{c}4.1 .2 \\
\text { (T04) }\end{array}$} & Mean & $2.1672 E+01$ & (1.0) & $2.1845 \mathrm{E}+01$ & $(4.0)$ & $2.2023 \mathrm{E}+01$ & $(5.0)$ & $2.1681 \mathrm{E}+01$ & $(2.0)$ & $2.1695 \mathrm{E}+01$ & $(3.0)$ \\
\hline & St. Dev. & $6.2420 \mathrm{E}-01$ & $=$ & 7.4503E-01 & $=$ & $8.0771 \mathrm{E}-01$ & $=$ & $1.4158 \mathrm{E}+00$ & $=$ & $1.6464 \mathrm{E}+00$ & --- \\
\hline \multicolumn{2}{|c|}{ Friedman Ave. Rank } & & 3.0 & & 4.0 & & 3.5 & & 2.5 & & 2.0 \\
\hline
\end{tabular}
Wilcoxon's rank sum test at $\alpha=0.05$. The results reveal that MGBDE/rand does as better as the other four DE variants on the selected two control problems.

According to the Friedman average rank and the Wilcoxon's rank sum test, it can be seen that the proposed MGBDE/rand outperforms MGBDE/bst-cur, GBDE, $\mathrm{DE} /$ best/1 and DE/rand/1.

Table 1. Comparisons of mean the best-of-run solutions 


\section{Conclusions}

Inspired by $\mathrm{DE} / \mathrm{rand} / 1$ and GBDE, this study proposed the so-called rand-type Gaussian mutation strategy to improve the search performance of MGBDE. The proposed Gaussian mutation strategy is also parameter free. This study uses the Friedman test and the Wilcoxon's signed rank test at $\alpha=0.05$ to compare the performance of multiple algorithms on two real-world optimal control problems given in IEEE - CEC 2011 evolutionary algorithm competition, viz., the bifunctional catalyst blend optimal control problem and the optimal control of a nonlinear stirred tank reactor,. Simulation results show that MGBDE/rand outperforms MGBDE/bst-cur, DE/rand/1, $\mathrm{DE} / \mathrm{best} / 1$, and GBDE in terms of solution accuracy.

\section{Acknowledgements}

This work was supported by the Ministry of Science and Technology, Taiwan, under Grant MOST 104-2221-E262-009.

\section{REFERENCES}

[1] R. Storn, K. Price. Differential evolution - a simple and efficient heuristic for global optimization over continuous space, J. Global Optim., Vol. 11, No. 4, 341-359, 1997.

[2] X. F. Lu, K. Tang, B. Sendhoff, X. Yao. A new self-adaptation scheme for differential evolution. Neurocomputing, Vol. 146, 2-16, 2014.

[3] J. Zhang, A. C. Sanderson. JADE: adaptive differential evolution with optional external archive, IEEE Trans. Evol. Comput., Vol. 13, No. 5, 945-958, 2009.

[4] S. M. Islam, S. Das, S. Ghosh, S. Roy, P. N. Suganthan, An adaptive differential evolution algorithm with novel mutation and crossover strategies for global numerical optimization, IEEE Trans. Syst., Man, Cybern.: B, Vol. 42,
No. 2, 482-500, 2012.

[5] J. Brest, S. Greiner, B. Bošković, M. Mernik, V. Žumer. Self-adapting control parameters in differential evolution: a comparative study on numerical benchmark problems, IEEE Trans. Evol. Comput., Vol. 10, No. 6, 646-657, 2006.

[6] M. G. H. Omran, A. P. Engelbrecht, A. Salman. Bare bones differential evolution, Eur. J. Oper. Res., Vol. 196, No. , 128-139, 2009.

[7] H. Wang, S. Rahnamayan, H. Sun, M. G. M. Omran. Gaussian bare-bones differential evolution, IEEE Trans. Cybern., Vol. 43, No. 2, 634-647, 2013.

[8] W. Gong, Z. Cai, X. Ling, H. Li. Enhanced differential evolution with adaptive strategies for numerical optimization, IEEE Trans. Syst., Man, Cybern.: B, Vol. 41, No. 2, 397-413, 2011.

[9] S. Das and P. N. Suganthan. Problem Definitions and Evaluation Criteria for CEC 2011 Competition on Testing Evolutionary Algorithms on Real World Optimization Problems, Nanyang Technol. Univ., Singapore, Tech. Rep., 2010.

[10] S. Garcia, A. Fernandez, J. Luengo, and F. Herrera, Advanced nonparametric tests for multiple comparisons in the design of experiments in computational intelligence and data mining: Experimental analysis of power, Information Science 180 (10) (2010) 2044-2064.

[11] J. Derrac, S. Garc'ia, D. Molina, F. Herrera, A practical tutorial on the use of nonparametric statistical tests as a methodology for comparing evolutionary and swarm intelligence algorithms, Swarm and Evolutionary Computation 1 (1) (2011) 3-18.

[12] R. Luus, B. Bojkov. Global optimization of the bifunctional catalyst problem, Can. J. Chem. Eng., Vol. 72, 160-163, 1994.

[13] R. Luus. Iterative Dynamic Programming, Chapman \& Hall/CRC Press, Boca Raton, FL, 2000.

[14] C .A. Floudas, P. M. Pardalos, C. S. Adjiman, W. R. Esposito, Z. H. Gumus, S.T. Harding, J. L. Klepeis, C. A. Meyer, C. A. Schweiger. Handbook of test problems in local and global optimization, Kluwer Academic Publishers, Dordrecht, The Netherlands, 1996. 